\title{
High Magnetic Field Effects on Plasma Wave THz Detection in Field-Effect Transistors
}

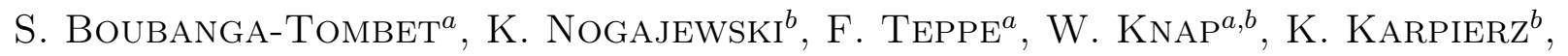 \\ J. ŁusAKOWSKI ${ }^{b}$, M. GRYNBERG ${ }^{b}$ AND M.I. DyAKONOV ${ }^{c}$ \\ ${ }^{a}$ Université Montpellier II and CNRS - GES - UMR5650, Place E. Bataillon, 34095 Montpellier, France \\ ${ }^{b}$ Institute of Experimental Physics, University of Warsaw, Hoża 69, 00-681 Warsaw, Poland \\ ${ }^{c}$ Laboratoire de Physique Théorique et Astroparticules, Université Montpellier II, CNRS \\ Place E. Bataillon, 34095 Montpellier, France
}

\begin{abstract}
Experiments on terahertz radiation detection with InGaAs/InAlAs field-effect transistor in quantizing magnetic field are reported. We observed oscillations of the photovoltaic signal analogous to the Shubnikov-de Haas oscillations, as well as their strong enhancement at the cyclotron resonance conditions. The results are described quantitatively within the frame of a theory which takes into account a new source of nonlinearity related to the Landau quantization of the conduction band.
\end{abstract}

PACS numbers: 07.57.Kp, 84.40.x, 85.30.Tv, 85.35.p, 73.20.Mf

\section{Introduction and background}

The observation of nonlinearities related to excitation of the plasma waves in a two-dimensional electron gas (2DEG) in field effect transistors (FETs) has opened a new route to create solid state high frequency devices $[1,2]$. These transistors can be used as selective $[3] \mathrm{THz}$ detectors at cryogenic temperatures, as well as broadband detectors at room temperature [4]. They were found to show a competitive noise equivalent power in comparison with other kinds of commercially available $\mathrm{THz}$ detectors $[5,6]$.

The detection signal results from the rectification of $\mathrm{THz}$ currents induced by the incident radiation in the transistor channel. The rectification takes place due to a nonlinear response of the gated 2DEG to the radiation. The main source of nonlinearity, explored until now, were changes in conductivity due to radiation-induced modulation of the carrier density in the channel. In this paper we discuss another source of nonlinearities i.e. changes in carrier mobility resulting from Landau quantization at high magnetic fields. The purpose of this work is to clarify the physical mechanism of Shubnikov-de Haas ( $\mathrm{SdH})$ oscillations and cyclotron resonance (CR) related detection by FETs. We investigate the $\mathrm{THz}$ response in a transistor of a simple architecture, with the gate covering the major part of the channel. In this structure, the effect of the gate bias on the observed signal can be clearly explained and the results can be compared with a recent theory [7].

\section{Results}

Experiments were carried out on a pseudomorphic InGaAs/InAlAs HEMT. The measurements were per- formed with an optically pumped molecular $\mathrm{CH}_{3} \mathrm{OH}$ laser. The radiation frequency was $2.5 \mathrm{THz}$. The photoconductivity signal (source to drain voltage) was measured using the standard lock-in technique.

Two types of experiments were performed. First, the detection signal was measured as a function of the gate voltage $V_{\mathrm{g}}$ at fixed magnetic field $B$. Second, the magnetic field was swept at a fixed gate voltage. The results are shown in Figs. 1 and 2. In both kinds of experiments the photoresponse shows an oscillatory behavior. Its periodicity versus $V_{\mathrm{g}}$ and $1 / B$ clearly indicates that the oscillations are related to the crossing between the Fermi level and the Landau levels (analogous to the SdH effect). Additionally, a strong enhancement of the signal is observed in magnetic fields close to cyclotron resonance magnetic field $B_{\mathrm{c}} \approx 4.5 \mathrm{~T}$.

Figure 1a shows experimental results of detection signal as a function of the gate voltage at different values of $B / B_{\mathrm{c}}(0.44,0.88,1,1.55)$. One can notice that the amplitude of oscillations grows as the magnetic field increases. This can be understood in terms of an increase of a nonlinearity related to $\mathrm{SdH}$ oscillations as described in Ref. [7] and [8]. In the post-cyclotron resonance region $\left(B>B_{\mathrm{c}}\right)$, the oscillations of the signal are damped. This can be qualitatively explained by considering the plasma wave dispersion law in the magnetic field: $k=\left(\omega^{2}-\omega_{\mathrm{c}}^{2}\right)^{1 / 2} / s$, where $s=\left(e U_{0} / m\right)^{1 / 2}$ is the plasma wave velocity at $B=0$. For $\omega_{\mathrm{c}}>\omega$ the plasma wave vector becomes imaginary, plasma waves cannot propagate, and one can expect a reduction of the signal, as evidenced in Fig. 1a.

Not only the oscillation amplitude of the signal, but also its background grows with $B$. By plotting the average value of the signal as a function of magnetic field, 

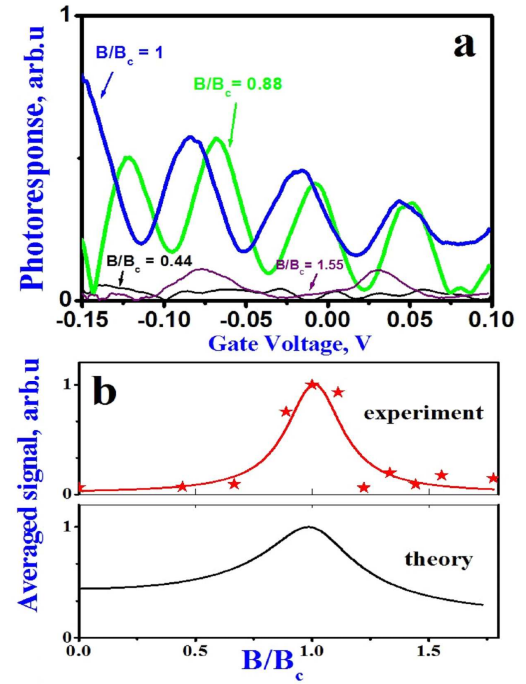

Fig. 1. (a) Experimental results of photoresponse at $2.5 \mathrm{THz}$ as a function of the gate voltage for different values of the magnetic field at $T=4.2 \mathrm{~K}$. Values of $B / B_{\mathrm{c}}$ are indicated. (b) The average signal as a function of magnetic field. Upper part: stars - experimental results; solid line — guide for the eyes. Lower part: calculations based on the model of Ref. [7].

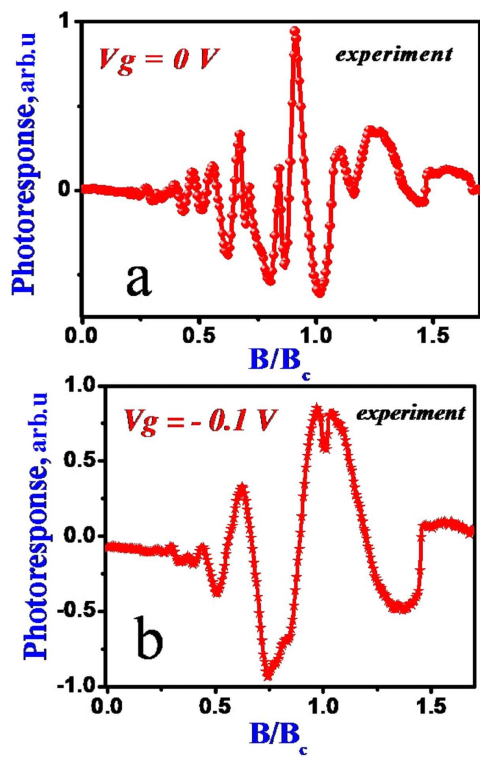

Fig. 2. (a) Experimental photoresponse as a function of the magnetic field for higher values of the electron density $\left(V_{\mathrm{g}}=0 \mathrm{~V}\right)$ at $4.2 \mathrm{~K}$. (b) The same as in (a) for a lower carrier density $\left(V_{\mathrm{g}}=-0.1 \mathrm{~V}\right)$.

one can see in Fig. 1b a pronounced maximum close to the CR condition. This enhancement can also be explained within the model of Ref. [7] (see the continuous line in Fig. 1b).

Figure 2 shows the measured magnetic field dependence for a fixed gate voltage (fixed electron density).
Figures $2 \mathrm{a}$ and $\mathrm{b}$ correspond to a higher and lower values of the electron density, respectively. For $B>B_{\mathrm{c}}$ the oscillation amplitude decreases. This is consistent with the results shown in Fig. 1b, and is also described by the theory [7].

\section{Conclusions}

In conclusion, we have performed magnetic field studies of plasma wave related detection in InGaAs/InAlAs field effect transistor. We observe oscillatory behavior of the photovoltaic signal similar to Shubnikov-de Haas oscillations, as well as a strong enhancement of the signal at the cyclotron resonance conditions. The experimental data are quantitatively reproduced by a theoretical model [7]. Our results clearly show that the detection mechanism is related to the $\mathrm{THz}$ rectification by nonlinearities of the plasma in the transistor channel.

\section{Acknowledgments}

The transistor used in this work was produced by the IEMN, Lille. We acknowledge the help of A. Cappy, S. Bollaert and A. Shchepetov. This work was supported by CNRS, the GDR-E project "Semiconductor sources and detectors of terahertz frequencies". We acknowledge the region of Languedoc-Roussillon through the "Terahertz Platform" project, the EU Grant No. MTKD-CT-2005-029671 and PL 162/THz/2006/02 program.

\section{References}

[1] M. Dyakonov, M.S. Shur, Phys. Rev. Lett. 71, 2465 (1993).

[2] M. Dyakonov, M.S. Shur, IEEE Trans. Electron. Dev. 43, 380 (1996).

[3] S. Boubanga-Tombet, F. Teppe, D. Coquillat, S. Nadar, N. Dyakonova, H. Videlier, W. Knap, A. Shchepetov, C. Gardès, Y. Roelens, S. Bollaert, D. Seliuta, R. Vadoklis, G. Valušis, Appl. Phys. Lett. 92, 212101 (2008).

[4] W. Knap, V. Kachorovskii, Y. Deng, S. Rumyantsev, J.Q. Lu, R. Gaska, M.S. Shur, G. Simin, X. Hu, M. Asif Khan, C.A. Saylor, L.C. Brunel, J. Appl. Phys. 91, 9346 (2002).

[5] R. Tauk, F. Teppe, S. Boubanga, D. Coquillat, W. Knap, Y.M. Meziani, C. Gallon, F. Boeuf, T. Skotnicki, C. Fenouillet-Beranger, D.K. Maude, S. Rumyantsev, M.S. Shur, Appl. Phys. Lett. $\mathbf{8 9}$ 253511 (2006).

[6] A. Lisauskas, W. von Spiegel, S. Boubanga-Tombet, A. El Fatimy, D. Coquillat, F. Teppe, N. Dyakonova, W. Knap, H.G. Roskos, Electron. Lett. 44, 408 (2008).

[7] M. Lifshits, M.I. Dyakonov, Phys. Rev. B 80, 121304(R) (2009).

[8] S. Boubanga-Tombet, M. Sakowicz, D. Coquillat, F. Teppe, W. Knap, M.I. Dyakonov, K. Karpierz, J. Łusakowski, M. Grynberg, Appl. Phys. Lett. 95, 072106 (2009). 